$\mathrm{J}$ o u r n a l of

Mathematics

and Applications

JMA No 40, pp 121-134 (2017)

\title{
On Some Qualitative Properties of Integrable Solutions for Cauchy-type Problem of Fractional Order
}

\author{
Mohamed M. A. Metwali
}

\begin{abstract}
The paper discusses the existence of solutions for Cauchytype problem of fractional order in the space of Lebesgue integrable functions on bounded interval. Some qualitative properties of solutions are presented such as monotonicity, uniqueness and continuous dependence on the initial data. The main tools used are measure of weak (strong) noncompactness, Darbo fixed point theorem and fractional calculus.
\end{abstract}

AMS Subject Classification: 45G10, 47H30, 47N20.

Keywords and Phrases: Cauchy problem; Darbo fixed point theorem; Quadratic integral equations; Measure of noncompactness.

\section{Introduction}

The differential equations involving Riemann-Liouville differential operators of fractional orders are widely used in modeling several physical phenomena (see e.g. $[22,23,25])$. The problems with this approach is in that they seem to deserve an independent study of their theory parallel to the well-known theory of ordinary differential equations.

The aim of this paper is to propose the solvability of the following nonlinear Cauchytype problem:

$$
\left\{\begin{array}{c}
D^{\alpha} u(t)=u(t) \cdot \int_{0}^{1} k(t, s) f(s, u(s)) d s, \quad \alpha \in(0,1), \\
u(0)=u_{0},
\end{array}\right.
$$

where $D^{\alpha}$ is the fractional derivative (in the sense of Riemann-Liouville) of order $\alpha$. The existence result for solutions to the hybrid differential equation

$$
\left\{\begin{array}{c}
\frac{d^{\alpha}}{d t^{\alpha}}[u(t)-f(t, u(t))]=g(t, u(t)), \text { a.e. } t \in\left[t_{0}, t_{0}+a\right], \\
u(0)=u_{0},
\end{array}\right.
$$

COPYRIGHT (C) by Publishing House of Rzeszów University of Technology P.O. Box 85, 35-959 Rzeszów, Poland 
has been discussed in [19] with the Riemann-Liouville fractional derivative and in [16] studied the existence of mild solutions for the above hybrid differential equation using the Caputo fractional derivative. This was extended by [24] to give approximation of solutions to Caputo fractional order hybrid differential equations.

The authors (see [15]) studied the Cauchy-type problem:

$$
\left\{\begin{array}{c}
D^{\alpha} u(t)=f(t, u(\varphi(t))), \\
\left.t^{1-\alpha} u(t)\right|_{t=0}=b, b>0,
\end{array}\right.
$$

where $D^{\alpha}$ is the Riemann-Liouville fractional derivative and the function $f(t, u)$ was assumed to be continuous. The existence of $L_{1}$-solution and investigated the behavior of solutions was proved in [12], where the operators were considered to be compact in $L_{p}$-spaces and this approach does not work for quadratic equations (see [10, Example $9.2]$ ). Our approach overcomes this difficulty.

Let us recall that the quadratic integral equations were discussed in many different functions spaces (see $[3,6,7,8,20]$ ) and have numerous applications in the theories of radiative transfer, neutron transport and in the kinetic theory of gases $[3,6,7]$.

Using the equivalence of the fractional Cauchy-type problem with the corresponding quadratic integral equation, we prove the existence of $L_{1}$-solution of equation (1.1).

Moreover, we discuss the monotonicity, uniqueness and Continuous dependence on the initial condition of the solution. To achieve our goal we use the technique of measure of weak (strong) noncompactness associated with Darbo fixed point theorem.

\section{Notation and auxiliary facts}

Let $\mathbb{R}$ be the field of real numbers, $J$ be the interval $[0,1]$ and $L_{1}(J)$ be the space of Lebesgue integrable functions (equivalence classes of functions) on a measurable subset $J$ of $\mathbb{R}$, with the standard norm

$$
\|x\|_{L_{1}(J)}=\int_{J}|x(t)| d t .
$$

By $L_{\infty}(J)$ we denote the Banach space of essentially bounded measurable functions with the essential supremum norm (denoted by $\|x\|_{L_{\infty}}$ ). We will write $L_{1}$ and $L_{\infty}$ instead of $L_{1}(J)$ and $L_{\infty}(J)$ respectively.

Definition 2.1. [2] Assume that a function $f: J \times \mathbb{R} \rightarrow \mathbb{R}$ satisfies the Carathéodory conditions i.e. it is measurable in $t$ for any $x \in \mathbb{R}$ and continuous in $x$ for almost all $t \in J$. Then to every function $x(t)$ being measurable on $J$ we assign

$$
(F x)(t)=f(t, x(t)), \quad t \in J .
$$

The operator $F$ is called the superposition (Nemytskii) operator. 
Theorem 2.1. [2] Let $f$ satisfies the Carathéodory conditions. The operator F maps continuously the space $L_{1}$ into itself if and only if

$$
|f(t, x)| \leq a(t)+q \cdot|x|
$$

for all $t \in J$ and $x \in \mathbb{R}$, where $a \in L_{1}$ and $q \geq 0$.

Let $S=S(J)$ denote the set of measurable (in Lebesgue sense) functions on $J$ and let meas stands for the Lebesgue measure in $\mathbb{R}$. Identifying the functions equal almost everywhere the set $S$ furnished with the metric

$$
d(x, y)=\inf _{a>0}[a+\operatorname{meas}\{s:|x(s)-y(s)| \geq a\}],
$$

becomes a complete metric space. Moreover, the convergence in measure on $J$ is equivalent to the convergence with respect to the metric $d$ (Proposition 2.14 in [26]). The compactness in such a space is called a "compactness in measure".

Theorem 2.2. [20] Let $X$ be a bounded subset of $L_{1}$ and suppose that there is a family of measurable subsets $\left(\Omega_{c}\right)_{0 \leq c \leq 1}$ of the interval $J$ such that meas $\Omega_{c}=c$ for every $c \in$ Jand for $x \in X$

$$
x\left(t_{1}\right) \geq x\left(t_{2}\right), \quad\left(t_{1} \in \Omega_{c}, \quad t_{2} \notin \Omega_{c}\right) .
$$

Then $X$ is compact in measure subset of $L_{1}$.

Theorem 2.3. [18, Theorem 6.2] The linear integral operator $K: L_{1} \rightarrow L_{1}$ given by a formula $(K u)(t)=\int_{J} k(t, s) u(s)$ ds preserve the monotonicity of functions iff

$$
\int_{0}^{l} k\left(t_{1}, s\right) d s \geq \int_{0}^{l} k\left(t_{2}, s\right) d s
$$

for $t_{1}<t_{2}, t_{1}, t_{2} \in J$ and for any $l \in J$.

Assume that $(E,\|\cdot\|)$ is an arbitrary Banach space with zero element $\theta$. Denote by $B(x, r)$ the closed ball centered at $x$ and with radius $r$. The symbol $B_{r}$ stands for the ball $B(\theta, r)$. Denote by $\mathcal{M}_{E}$ the family of all nonempty and bounded subsets of $E$ and by $\mathcal{N}_{E}, \mathcal{N}_{E}^{W}$ its subfamilies consisting of all relatively compact and weakly relatively compact sets, respectively. The symbols $\bar{X}, \bar{X}^{W}$ stand for the closure and the weak closure of a set $\mathrm{X}$, respectively and $\operatorname{conv} X$ will denote the convex closure of $X$.

Definition 2.2. [4] A mapping $\mu: \mathcal{M}_{E} \rightarrow[0, \infty)$ is called a regular measure of noncompactness in $E$ if the following conditions hold:

(i) $\mu(X)=0 \Leftrightarrow X \in \mathcal{N}_{E}$.

(ii) $X \subset Y \Rightarrow \mu(X) \leq \mu(Y)$.

(iii) $\mu(\bar{X})=\mu(\operatorname{conv} X)=\mu(X)$. 
(iv) $\mu(\lambda X)=|\lambda| \mu(X)$, for $\lambda \in \mathbb{R}$.

(v) $\mu(X+Y) \leq \mu(X)+\mu(Y)$.

(vi) $\mu(X \cup Y)=\max \{\mu(X), \mu(Y)\}$.

(vii) If $X_{n}$ is a sequence of nonempty, bounded, closed subsets of $E, X_{n}=\bar{X}_{n}$ such that $X_{n+1} \subset X_{n}, n=1,2,3, \ldots$, and $\lim _{n \rightarrow \infty} \mu\left(X_{n}\right)=0$, then the set $X_{\infty}=\bigcap_{n=1}^{\infty} X_{n}$ is nonempty.

An example of such a mapping is the following:

Definition 2.3. [4] Let $X$ be a nonempty and bounded subset of $E$. The Hausdorff measure of noncompactness $\chi(X)$ is defined as

$\chi(X)=\inf \left\{r>0:\right.$ there exists a finite subset $\mathrm{Y}$ of $\mathrm{E}$ such that $\left.x \subset Y+B_{r}\right\}$.

Definition 2.4. [4] A mapping $\mu: \mathcal{M}_{E} \rightarrow[0, \infty)$ is called a regular measure of weak noncompactness in $E$ if it satisfies conditions (ii)-(vi) of Definition 2.2 and the following two conditions (being counterparts of (i) and (vii)) hold:

(i') $\mu(X)=0 \Leftrightarrow X \in \mathcal{N}_{E}^{W}$.

(vii') If $X_{n}$ is a sequence of nonempty, bounded, closed subsets of $E, X_{n}=\bar{X}_{n}{ }^{W}$ such that $X_{n+1} \subset X_{n}, n=1,2,3, \ldots$, and $\lim _{n \rightarrow \infty} \mu\left(X_{n}\right)=0$, then the set $X_{\infty}=\bigcap_{n=1}^{\infty} X_{n}$ is nonempty.

Another regular measure was defined in the space $L_{1}$ (cf. [5]). For any $\varepsilon>0$, let $c$ be a measure of equiintegrability of the set $X[2$, p. 39] i.e.

$$
c(X)=\lim _{\varepsilon \rightarrow 0}\left\{\sup _{x \in X}\left\{\sup \left[\int_{D}|x(t)| d t, D \subset J, \text { meas } D \leq \varepsilon\right]\right\}\right\} .
$$

It forms a regular measure of noncompactness if restricted to the family of subsets being compact in measure (cf. [14]).

Theorem 2.4. [4] Let $Q$ be a nonempty, bounded, closed, and convex subset of $E$ and let $H: Q \rightarrow Q$ be a continuous transformation which is a contraction with respect to the measure of noncompactness $\mu$, i.e. there exists $\gamma \in[0,1)$ such that

$$
\mu(H(X)) \leq \gamma \mu(X),
$$

for any nonempty subset $X$ of $E$. Then $H$ has at least one fixed point in the set $Q$.

Next, we introduce a short note about fractional calculus theory.

Definition 2.5. [17] The Riemann-Liouville fractional derivative of order $\alpha>0$ of a continuous function $f:(0, \infty) \rightarrow \mathbb{R}$ is given by

$$
D^{\alpha} f(t)=\frac{1}{\Gamma(n-\alpha)} \frac{d^{n}}{d t^{n}} \int_{0}^{t} \frac{f(s)}{(t-s)^{\alpha-n+1}} d s
$$

provided that the right side is pointwise defined on $(0, \infty)$, where $n=[\alpha]+1$ and $[\alpha]$ denotes the integer part of $\alpha$. 
Definition 2.6. [17] The Riemann-Liouville fractional integral of order $\alpha>0$ of a function $f:(0, \infty) \rightarrow \mathbb{R}$ is defined by

$$
I^{\alpha} f(t)=\frac{1}{\Gamma(\alpha)} \int_{0}^{t} \frac{f(s)}{(t-s)^{1-\alpha}} d s
$$

provided that the right side is pointwise defined on $(0, \infty)$.

Lemma 2.1. [13, 17]. Let $f \in L_{1}$ and let $\alpha \in(0,1)$, then

(a) $D^{\alpha} I^{\alpha} f(t)=f(t)$

(b) $I^{\alpha} D^{\alpha} f(t)=f(t)-f(0)$.

(c) The operator $I^{\alpha}$ maps $L_{1}$ into itself continuously.

(d) The operator $I^{\alpha}$ maps the monotonic nondecreasing function into functions of the same type.

\section{Main result}

First we need to prove the equivalence of (1.1) with the corresponding quadratic integral equation:

$$
u(t)=u_{0}+\int_{0}^{t} \frac{(t-s)^{\alpha-1}}{\Gamma(\alpha)}\left(u(s) \cdot \int_{0}^{1} k(s, v) f(v, u(v)) d v\right) d s, \quad t \in(0,1)
$$

Indeed, let $u(t)$ be a solution of (1.1). Applying the operator $I^{\alpha}$ to both sides of (1.1). By Lemma 2.1, we have

$$
I^{\alpha} D^{\alpha} u(t)=u(t)-u(0)=I^{\alpha}\left(u(t) \cdot \int_{0}^{1} k(t, s) f(s, u(s)) d s\right),
$$

where $u(0)=u_{0}$, we have

$$
u(t)=u_{0}+\int_{0}^{t} \frac{(t-s)^{\alpha-1}}{\Gamma(\alpha)}\left(u(s) \cdot \int_{0}^{1} k(s, v) f(v, u(v)) d v\right) d s .
$$

Thus equation (3.1) holds.

Conversely, let $u(t)$ be a solution of equation (3.1). Then applying $D^{\alpha}$ on both sides of (3.1), we obtain (1.1). Finally, put $t=0$ in equation $(3.1)$, we get $u(0)=u_{0}$. Then problem (1.1) and equation (3.1) are equivalent to each other. 


\subsection{Existence of monotonic integrable solution}

Rewrite (3.1) as

$$
u=T u
$$

where

$$
T u(t)=u_{0}+I^{\alpha}(A u)(t),(A u)(t)=u(t) \cdot(K F u)(t),
$$

$(K u)(t)=\int_{0}^{1} k(t, s) u(s) d s, F u=f(t, u)$, and $I^{\alpha}$ is as in Definition 2.6.

To solve equation (3.1) it is necessary to find a fixed point of the operator $T$. For facilitating our discussion, we shall treat (3.1) under the following assumptions listed below:

(i) $f: J \times \mathbb{R} \rightarrow \mathbb{R}$ satisfies Carathéodory conditions and $f(t, u) \geq 0$ for $u \geq 0$. Moreover, $f$ is assumed to be nondecreasing with respect to both variables $t$ and $u$ separately.

(ii) There is a positive function $a \in L_{1}$ and a constant $q \geq 0$ such that

$$
|f(t, u)| \leq a(t)+q|u|
$$

for all $t \in J$ and $u \in \mathbb{R}$.

(iii) $k(t, s): J \times J \rightarrow \mathbb{R}$ is measurable with respect to both variables. The linear integral operator $K$ associated with the kernel $k$ maps $L_{1}$ into $L_{\infty}$ and is continuous.

(iv) For any $0<l<1$ the following condition holds true

$$
t_{1}<t_{2} \Rightarrow \int_{0}^{l} k\left(t_{1}, s\right) d s \geq \int_{0}^{l} k\left(t_{2}, s\right) d s .
$$

(v) Let $r>0$ be such that $\|K\|_{L_{\infty}}\left(\|a\|_{L_{1}}+q \cdot r\right)<\Gamma(\alpha+1)$.

Theorem 3.1. Let assumptions (i)-(v) be satisfied, then the Cauchy-type problem (1.1) has at least one solution in $L_{1}$ a.e. nondecreasing on $J$.

Proof. First of all observe that by assumptions (i), (ii) and Theorem 2.1 we have that the superposition operator $F$ maps continuously $L_{1}$ into itself. From assumption (iii) the operator $(K F)$ maps $L_{1}$ into $L_{\infty}$. From the Hölder inequality the operator $A$ maps $L_{1}$ into itself and is continuous. Finally, since the operator $I^{\alpha}$ maps $L_{1}$ into itself continuously, then we can deduce that the operator $T$ maps $L_{1}$ into itself and is continuous. 
Thus for $u \in L_{1}$, we have

$$
\begin{aligned}
\|T u\|_{L_{1}} & =\int_{0}^{1}|(T u)(t)| d t \leq \int_{0}^{1} u_{0} d t+\int_{0}^{1} I^{\alpha}(A u)(t) d t \\
& \leq\left. u_{0} \cdot t\right|_{0} ^{1}+\int_{0}^{1}\left|\int_{0}^{t} \frac{(t-s)^{\alpha-1}}{\Gamma(\alpha)}\left(u(s) \cdot \int_{0}^{1} k(s, v) f(v, u(v)) d v\right) d s\right| d t \\
& \leq u_{0}+\int_{0}^{1}\left|\int_{0}^{t} \frac{(t-s)^{\alpha-1}}{\Gamma(\alpha)}\left(u(s) \cdot \int_{0}^{1} k(s, v)(a(v)+q|u(v)|) d v\right) d s\right| d t \\
& \leq u_{0}+\int_{0}^{1}\left|\int_{0}^{t} \frac{(t-s)^{\alpha-1}}{\Gamma(\alpha)} u(s) \cdot\|K\|_{L_{\infty}}\left(\|a\|_{L_{1}}+q\|u\|_{L_{1}}\right) d s\right| d t \\
& \leq u_{0}+\|K\|_{L_{\infty}}\left(\|a\|_{L_{1}}+q\|u\|_{L_{1}}\right) \int_{0}^{1} \frac{t^{\alpha-1}}{\Gamma(\alpha)} d t \int_{0}^{1}|u(s)| d s \\
& \leq u_{0}+\left.\|K\|_{L_{\infty}}\left(\|a\|_{L_{1}}+q\|u\|_{L_{1}}\right) \frac{t^{\alpha}}{\alpha \Gamma(\alpha)}\right|_{0} ^{1} \cdot\|u\|_{L_{1}} \\
& =u_{0}+\frac{\|K\|_{L_{\infty}}}{\Gamma(\alpha+1)}\left(\|a\|_{L_{1}}+q\|u\|_{L_{1}}\right) \cdot\|u\|_{L_{1}},
\end{aligned}
$$

so the function $T u$ is bounded in $J$. This allows us to infer that the operator $T$ transforms $L_{1}$ into itself. Moreover, this estimate the following

$$
\|T u\|_{L_{1}} \leq u_{0}+\frac{\|K\|_{L_{\infty}}}{\Gamma(\alpha+1)}\left(\|a\|_{L_{1}}+q \cdot r\right) \cdot r \leq r .
$$

As a domain for the operator $T$ we will consider the ball $B_{r}$, where $r$ is the positive solution of the equation

$$
u_{0}+\frac{\|K\|_{L_{\infty}}}{\Gamma(\alpha+1)}\left(\|a\|_{L_{1}}+q \cdot r\right) \cdot r=r
$$

Let us remark, that the above inequality is of the form $\hat{a}+(\hat{b}+\hat{v} r) \hat{c} r \leq r$ with $\hat{a}, \hat{b}, \hat{c}, \hat{v}>0$. Then $\hat{v} \hat{c}>0$ and by assumption (v), we have that $\hat{b} \hat{c}-1<0$ and that the discriminant is positive, then Viète's formulas imply that the quadratic equation has two positive solutions $r_{1}<r_{2}$. So there exists a positive number $r>0$ satisfying this inequality such that $T$ maps the ball $B_{r}$ into itself and is continuous.

Further, let $Q_{r}$ is a subset of $B_{r}$ which has the functions a.e. nondecreasing on $J$. This set is nonempty, bounded (by $r$ ), convex and closed in $L_{1}$. In view of Theorem 2.2 the set $Q_{r}$ is compact in measure.

Now, we will show that $T$ preserve the monotonicity of functions. Take $u \in Q_{r}$, then $u(t)$ is a.e. nondecreasing on $J$ and consequently $F$ is also of the same type by virtue of the assumption (i). Further, $K u(t)$ is a.e. nondecreasing on $J$ due to assumption (iv). Since the pointwise product of a.e. monotone functions is still of the same type, the operator $A=u \cdot(K F u)$ is a.e. nondecreasing on $J$. Moreover, the operator $I^{\alpha}$ maps a.e. nondecreasing functions into functions of the same type 
(thanks to Lemma 2.1). Thus we can deduce that $T u$ is also a.e. nondecreasing on $J$. Then $T$ maps continuously $Q_{r}$ into $Q_{r}$.

From now we will assume that $X$ is a nonempty subset of $Q_{r}$ and the constant $\varepsilon>0$ is arbitrary, but fixed. Then for an arbitrary $u \in X$ and for a set $D \subset J$, meas $D \leq \varepsilon$ we obtain

$$
\begin{aligned}
\|T u\|_{L_{1}(D)}= & \int_{D}|T u(t)| d t \leq \int_{J} u_{0} \cdot \chi_{D}(t) d t+\int_{D} I^{\alpha}(A u)(t) d t \\
\leq & \left\|u_{0} \cdot \chi_{D}\right\|_{L_{1}} \\
& \quad+\int_{D}\left|\int_{0}^{t} \frac{(t-s)^{\alpha-1}}{\Gamma(\alpha)}\left(u(s) \cdot \int_{0}^{1} k(s, v) f(v, u(v)) d v\right) d s\right| d t \\
\leq & \left\|u_{0} \cdot \chi_{D}\right\|_{L_{1}} \\
& \quad+\int_{D}\left|\int_{0}^{t} \frac{(t-s)^{\alpha-1}}{\Gamma(\alpha)}\left(u(s) \cdot\|K\|_{L_{\infty}}\left[\|a\|_{L_{1}}+q\|u\|_{L_{1}}\right]\right) d s\right| d t \\
\leq & \left\|u_{0} \cdot \chi_{D}\right\|_{L_{1}}+\|K\|_{L_{\infty}}\left(\|a\|_{L_{1}}+q \cdot r\right) \int_{0}^{1} \frac{t^{\alpha-1}}{\Gamma(\alpha)} d t \cdot \int_{D}|u(s)| d s \\
\leq & \left\|u_{0} \cdot \chi_{D}\right\|_{L_{1}}+\frac{\|K\|_{L_{\infty}}\left(\|a\|_{L_{1}}+q \cdot r\right)}{\Gamma(\alpha+1)} \cdot \int_{D}|u(s)| d s .
\end{aligned}
$$

Hence, taking into account the equality

$$
\lim _{\varepsilon \rightarrow 0}\left\{\sup \left[\left\|u_{0} \cdot \chi_{D}\right\|_{L_{1}}: D \subset J, \text { meas } D \leq \varepsilon\right]\right\}=0,
$$

and by the definition of $c(X)$, we get

$$
c(T X) \leq \frac{\|K\|_{L_{\infty}}\left(\|a\|_{L_{1}}+q \cdot r\right)}{\Gamma(\alpha+1)} \cdot c(X) .
$$

Recall that $\|K\|_{L_{\infty}}\left(\|a\|_{L_{1}}+q \cdot r\right)<\Gamma(\alpha+1)$ and the inequality obtained above with the properties of the operator $T$ and since the set $Q_{r}$ is compact in measure we are able to apply Theorem 2.4 which completes the proof.

\subsection{Uniqueness of the solution}

We will discuss the uniqueness of solutions in the following theorem:

Theorem 3.2. Let assumptions of Theorem 3.1 be satisfied, but instead of assumptions (ii) and (v) consider the following condition:

(vi) Assume that, there is a constant $q \geq 0$ such that for a.e. $t \in J$

$$
|f(t, u)-f(t, v)| \leq q|u-v|, \text { and }|f(t, 0)| \leq a(t),
$$

where $u, v \in \mathbb{R}$ and $a \in L_{1}$.

Moreover, let

$$
\left(\frac{\|K\|_{L_{\infty}}\left(\|a\|_{L_{1}}+2 q \cdot r\right)}{\Gamma(\alpha+1)}\right)<1 .
$$


Then the Cauchy-type problem (1.1) has an unique solution in $L_{1}(J)$.

Proof. From assumption (vi), we have

$$
\begin{aligned}
|| f(t, u)|-| f(t, 0)|| & \leq|f(t, u)-f(t, 0)| \leq q|u| \\
\Rightarrow|f(t, u)| & \leq|f(t, 0)|+q|u| \leq a(t)+q|u|,
\end{aligned}
$$

which imply that assumption (ii) and (v) of Theorem 1.1 are satisfied.

For the uniqueness solution of (1.1), let $x(t)$ and $y(t)$ be any two solutions of (3.1) in $B_{r}$, then we have

$$
\begin{aligned}
|x(t)-y(t)| \leq & \mid I^{\alpha}\left(x(t) \cdot \int_{0}^{1} k(t, v) f(v, x(v)) d v\right) \\
& -I^{\alpha}\left(y(t) \cdot \int_{0}^{1} k(t, v) f(v, y(v)) d v\right) \mid \\
\leq & \mid I^{\alpha}\left(x(t) \cdot \int_{0}^{1} k(t, v) f(v, x(v)) d v\right) \\
& -I^{\alpha}\left(x(t) \cdot \int_{0}^{1} k(t, v) f(v, y(v)) d v\right) \mid \\
& +\mid I^{\alpha}\left(x(t) \cdot \int_{0}^{1} k(t, v) f(v, y(v)) d v\right) \\
& -I^{\alpha}\left(y(t) \cdot \int_{0}^{1} k(t, v) f(v, y(v)) d v\right) \mid \\
\leq & I^{\alpha}\left(|x(t)| \cdot \int_{0}^{1}|k(t, v)||f(v, x(v))-f(v, y(v))| d v\right) \\
& +I^{\alpha}\left(|x(t)-y(t)| \cdot \int_{0}^{1}|k(t, v)||f(v, y(v))| d v\right) \\
\leq & I^{\alpha}\left(|x(t)| \cdot \int_{0}^{1}|k(t, v)| q|x(v)-y(v)| d v\right) \\
& +I^{\alpha}\left(|x(t)-y(t)| \cdot \int_{0}^{1}|k(t, v)|[a(v)+q \mid y(v)) \mid d v\right) .
\end{aligned}
$$

Therefore,

$$
\begin{aligned}
\|x-y\|_{L_{1}} \leq & \int_{0}^{1}\left\{\int_{0}^{t} \frac{(t-s)^{\alpha-1}}{\Gamma(\alpha)}\left(|x(s)| \cdot \int_{0}^{1}|k(s, v)| q|x(v)-y(v)| d v\right) d s\right\} d t \\
& +\int_{0}^{1}\left\{\int_{0}^{t} \frac{(t-s)^{\alpha-1}}{\Gamma(\alpha)}\right. \\
& \left(|x(s)-y(s)| \cdot \int_{0}^{1}|k(s, v)|[a(v)+q|y(v)| d v) d s\right\} d t
\end{aligned}
$$




$$
\begin{aligned}
& \leq \frac{\|x\|_{L_{1}} \cdot q\|K\|_{L_{\infty}}}{\Gamma(\alpha+1)} \cdot\|x-y\|_{L_{1}}+\frac{\|K\|_{L_{\infty}}\left(\|a\|_{L_{1}}+q\|y\|_{L_{1}}\right)}{\Gamma(\alpha+1)} \cdot\|x-y\|_{L_{1}} \\
& \leq\left(\frac{q \cdot r \cdot\|K\|_{\infty}+\|K\|_{L_{\infty}}\left(\|a\|_{L_{1}}+q \cdot r\right)}{\Gamma(\alpha+1)}\right) \cdot\|x-y\|_{L_{1}} .
\end{aligned}
$$

The above inequality yields

$$
\left(1-\frac{\|K\|_{L_{\infty}}\left(\|a\|_{L_{1}}+2 q \cdot r\right)}{\Gamma(\alpha+1)}\right) \cdot\|x-y\|_{L_{1}} \leq 0
$$

which implies that

$$
\|x-y\|_{L_{1}}=0 \Rightarrow x=y .
$$

This complete the proof.

\subsection{Continuous dependence on the initial condition}

In the present section sufficient conditions are obtained under which the solution $u\left(t, 0, u_{0}\right)$ of problem (1.1) depends continuously on the initial conditions.

Definition 3.1. [11] We say that the solution $u\left(t, 0, u_{0}\right)$ of problem (1.1) depends continuously on the initial conditions for $t \in J$, if for every two positive numbers $\epsilon$ and $\eta$ there exists a number $\delta=\delta(\epsilon, \eta)>0$ such that if $\left|u_{0}-\tilde{u}_{0}\right|<\delta$ then $\left\|u\left(t, 0, u_{0}\right)-\tilde{u}\left(t, 0, \tilde{u_{0}}\right)\right\|_{L_{1}}<\epsilon$ for $t \in J$ and $|t|>\eta$.

Theorem 3.3. Let assumptions of Theorem 3.2 be satisfied, then the solution of the Cauchy-type problem (1.1) depends continuously on the initial condition in J.

Proof. According to Theorem 3.2, the problem (1.1) has an unique solution. We will prove that this solution depends continuously on the initial condition in $J$.

Let $u(t)$ be a solution of

$$
u(t)=u_{0}+\int_{0}^{t} \frac{(t-s)^{\alpha-1}}{\Gamma(\alpha)}\left(u(s) \cdot \int_{0}^{1} k(s, v) f(v, u(v)) d v\right) d s
$$


and let $\tilde{u}(t)$ be a solution of the above equation such that $\tilde{u}(0)=\tilde{u_{0}}$, then

$$
\begin{aligned}
|u(t)-\tilde{u}(t)|= & \left|u_{0}-\tilde{u_{0}}\right| \\
& +\mid I^{\alpha}\left(u(t) \cdot \int_{0}^{1} k(t, v) f(v, u(v)) d v\right) d s \\
& -I^{\alpha}\left(\tilde{u}(t) \cdot \int_{0}^{1} k(t, v) f(v, \tilde{u}(v)) d v\right) d s \mid \\
\leq & \left|u_{0}-\tilde{u_{0}}\right|+I^{\alpha}\left(|u(t)| \cdot \int_{0}^{1}|k(t, v)||f(v, u(v))-f(v, \tilde{u}(v))| d v\right) \\
& +I^{\alpha}\left(|u(t)-\tilde{u}(t)| \cdot \int_{0}^{1}|k(t, v)||f(v, \tilde{u}(v))| d v\right) \\
\leq & \left|u_{0}-\tilde{u_{0}}\right|+I^{\alpha}\left(|u(t)| \cdot \int_{0}^{1}|k(t, v)| q|u(v)-\tilde{u}(v)| d v\right) \\
& +I^{\alpha}\left(|u(t)-\tilde{u}(t)| \cdot \int_{0}^{1}|k(t, v)|[|a(v)+q| \tilde{u}(v) \mid] d v\right) .
\end{aligned}
$$

Therefore,

$$
\begin{aligned}
\|u-\tilde{u}\|_{L_{1}} & \leq \int_{0}^{1}\left|u_{0}-\tilde{u_{0}}\right| d t \\
& +\int_{0}^{1}\left\{\int_{0}^{t} \frac{(t-s)^{\alpha-1}}{\Gamma(\alpha)}\left(|u(s)| \cdot \int_{0}^{1}|k(s, v)| q|u(v)-\tilde{u}(v)| d v\right) d s\right\} d t \\
& +\int_{0}^{1}\left\{\int_{0}^{t} \frac{(t-s)^{\alpha-1}}{\Gamma(\alpha)}\right. \\
& \left.\left(|u(s)-\tilde{u}(s)| \cdot \int_{0}^{1}|k(s, v)|[|a(v)+q| \tilde{u}(v) \mid] d v\right) d s\right\} d t \\
\leq & \left|u_{0}-\tilde{u_{0}}\right|+\left(\frac{q \cdot r \cdot\|K\|_{\infty}+\|K\|_{L_{\infty}}\left(\|a\|_{L_{1}}+q \cdot r\right)}{\Gamma(\alpha+1)}\right) \cdot\|u-\tilde{u}\|_{L_{1}} .
\end{aligned}
$$

Then we get

$$
\|u-\tilde{u}\|_{L_{1}} \leq\left(1-\frac{\|K\|_{L_{\infty}}\left(\|a\|_{L_{1}}+2 q \cdot r\right)}{\Gamma(\alpha+1)}\right)^{-1} \cdot\left|u_{0}-\tilde{u_{0}}\right| .
$$

Therefore, if $\left|u_{0}-\tilde{u_{0}}\right|<\delta(\epsilon)$, then $\|u-\tilde{u}\|_{L_{1}}<\epsilon$, where

$$
\delta(\epsilon)=\epsilon \cdot \frac{\Gamma(\alpha+1)-\|K\|_{L_{\infty}}\left(\|a\|_{L_{1}}+2 q \cdot r\right)}{\Gamma(\alpha+1)} .
$$

Now from the equivalence of (1.1) and (3.1), we get that the solution of the Cauchytype problem (1.1) depends continuously on the initial condition in $J$. 


\subsection{Remarks}

We need to stress on some aspects of obtained results. First of all we can observe, that our solutions are not necessarily continuous as in almost all previously investigated cases (cf. $[1,21])$. So we need not to assume, that the Hammerstein operator transforms the space $C(I)$ into itself. Our solutions belong to the space $L_{1}$, for the examples and conditions related to Hammerstein operators in $L_{1}$ we refer the readers to $[20,27]$.

We need to emphasize that the following Cauchy-type problem is also strictly related to quadratic equations (cf. [8])

$$
\left(\frac{x(t)-g(t)}{f_{1}(t, x)}\right)^{\prime}=f_{2}(t, x(t)), \quad x(0)=0,
$$

where $f_{1}: I \times \mathbb{R} \rightarrow \mathbb{R} \backslash\{0\}$.

It can be easily checked that under some typical assumptions this problem is equivalent to the integral equation [9]

$$
x(t)=g(t)+f_{1}(t, x(t)) \cdot\left(\int_{0}^{t} f_{2}(s, x(s)) d s-\frac{g(0)}{f_{1}(0,0)}\right) .
$$

Nevertheless, when we are looking for continuous solutions for integral equation, for differential one we obtain classical solutions, i.e. $x$ is continuously differentiable. In the case presented above we investigate Carathéodory solutions for the Cauchy problem.

\section{References}

[1] A. Aghajani, M. Aliaskari and A. Shole Haghighi, Some existence theorems for systems of equations involving condensing operators and applications, Mediterr. J. Math. (2017) 14-47.

[2] J. Appell, P.P. Zabrejko, Nonlinear Superposition Operators, Cambridge Tracts in Mathematics 95, Cambridge University Press, Cambridge 1990.

[3] I.K. Argyros, On a class of quadratic integral equations with perturbations, Functiones et Approximatio 20 (1992) 51-63.

[4] J. Banaś, K. Goebel, Measures of Noncompactness in Banach Spaces, Lect. Notes in Math. 60, M. Dekker, New York-Basel 1980.

[5] J. Banaś, L. Olszowy, Measures of noncompactness related to monotonicity, Comment. Math. Prace Matem. 41 (2001) 13-23.

[6] J. Banaś, K. Sadarangani, Solutions of some functional-integral equations in Banach algebras, Math. Comput. Model. 38 (2003) 245-250. 
[7] J. Caballero, A.B. Mingarelli and K. Sadarangani, Existence of solutions of an integral equation of Chandrasekhar type in the theory of radiative transfer, Electr. Jour. Differ. Equat. 57 (2006) 1-11.

[8] M. Cichoń, M. Metwali, On a fixed point theorem for the product of operators, J. Fixed Point Theory Appl. 18 (2016) 753-770.

[9] M. Cichoń, M. Metwali, On the existence of solutions for quadratic integral equations in Orlicz spaces, Math. Slovaca 66 (2016) 1413-1426.

[10] K. Deimling, Nonlinear Functional Analysis, Springer, Berlin 1985.

[11] A. Dishlev, D. Bainov, Continuous dependence on the initial condition of the solution of a system of differential equations with variable structure and with impulses, Putt. RIMS, Kyoto Univ. 23 (1987) 923-936.

[12] A.M.A. El-Sayed, Sh.A. Abd El-Salam, Weighted Cauchy-type problem of a functional differ-integral equation, Electronic Journal of Qualitative Theory of Differential Equations 30 (2007) 1-9.

[13] A.M.A. El-Sayed, Nonlinear functional differential equations of arbitrary order, Nonlin. Anal. 33 (1998) 181-186.

[14] N. Erzakova, Compactness in measure and measure of noncompactness, Siberian Math. J. 38 (1997) 926-928.

[15] K.M. Furati, N.E. Tatar, Power-type estimates for a nonlinear fractional differential equation, Nonlin. Anal. 62 (2005) 1025-1036.

[16] M.A.E. Herzallah, D. Baleanu, On fractional order hybrid differential equations, Abstr. Appl. Anal., Volume 2014 (2014), Article ID 389386, 7 pages, doi:10.1155/2014/389386.

[17] A.A. Kilbas, H.M. Srivastava and J.J. Trujillo, Theory and Applications of Fractional Differential Equations, Elsevier Science Inc., New York, NY, USA 2006.

[18] J. Krzyż, On monotonicity-preserving transformations, Ann. UMCS 6 (1952) 91-111.

[19] H. Lu, S. Sun, D. Yang and H. Teng, Theory of fractional hybrid differential equations with linear perturbations of second type, Bound. Value Probl. (2013) 2013:23 16 pages.

[20] M. Metwali, On a class of quadratic Urysohn-Hammerstein integral equations of mixed type and initial value problem of fractional order, Mediterr. J. Math. 13 (2016) 2691-2707.

[21] L. Mishra, M. Sen and R. Mohapatra, On Existence theorems for some generalized nonlinear functional-integral equations with applications, Filomat 31 (2017) 2081-2091. 
[22] I. Podlubny, Fractional Differential Equations, Acad. Press, San Diego-New York-London 1999.

[23] Yu. Rossikhin, M.V. Shitikova, Applications of fractional calculus to dynamic problems of linear and nonlinear hereditary mechanics of solids, Appl. Mech. Rev. 50 (1997) 15-67.

[24] D. Somjaiwang, P. Sa Ngiamsunthorn, Existence and approximation of solutions to fractional order hybrid differential equations, Advances in Difference Equations (2016) 2016-278.

[25] T.A. Surguladze, On certain applications of fractional calculus to viscoelasticity, J. Math. Sci. 112 (2002) 4517-4525.

[26] M. Väth, Volterra and Integral Equations of Vector Functions, Marcel Dekker, New York-Basel 2000.

[27] P.P. Zabrejko, A.I. Koshlev, M.A. Krasnoselskii, S.G. Mikhlin, L.S. Rakovshchik and V.J. Stecenko, Integral Equations, Noordhoff, Leyden 1975.

\section{DOI: $10.7862 / \mathrm{rf} .2017 .8$}

Mohamed M.A. Metwali email: m.metwali@yahoo.com

Department of Mathematics Faculty of Sciences

Damanhour University

EGYPT

Received 6.10.2017 